\title{
الخطء لخط الطول أولغ بك في الزيج الجديد السلطاني
}

\author{
Ihsan Kamil Lathif \\ UIN Walisanga Semarang \\ (Email:Ihsan.elhasany@gmail.com)
}

\begin{abstract}
ملخص

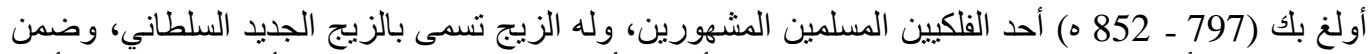

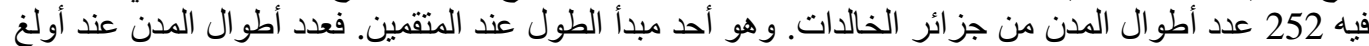

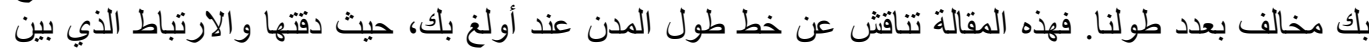
طول المدن أولغ بك و الطول الجرينتشية. واستخدمنا في تشريح مناقتشة النوعية المكتبية (Research Library) باستفادة المنهج التاريخي (Historical Approach), وفي تحليل البيل البيانات التحليل النوعي. والنيل المضمون (content analisis)

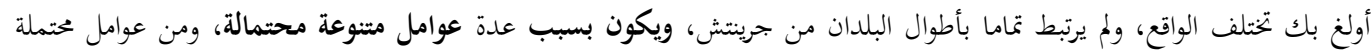

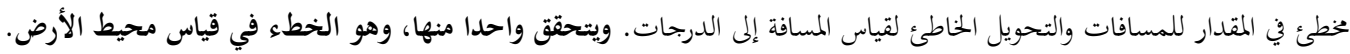

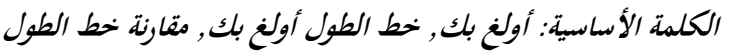

معظم الحساب، حيث خالف الواقع، فانرف سمت القبلة من النقطة الفعلية وكذا غيره، ولذا كانت معرفة دقة أطواله تشرح لنا خزنة من خزائن العلماء المتقدمين وتوضح تطور عدد الأطوال من قترة أولغ بك إلى وقتنا الحاضر

فالتناقش في هذه المقالة، إظهار الخطء في خط الطول أولغ بك وإعادة حسابه، والبحث السابق لمن تقدم لم يظهر الخطء في خط الطول أولغ بك، ومعظم الباحثين السابقين يتناقش ما يتعلق باحداثيات الجغرافية لبطليموس (ت. 170 م), حيث دقتها وخطئها. وأما إ. س كينيدي و ح. ح.م. (E. S. Kennedy and M. H. Regier ${ }^{4}$ ) فيجيه فيظهر العلاقات الخطية لعدد خط الطول عند العلماء المسلمين في العصور الوسطى على الجملة. ونتيجته أن

${ }^{4}$ E. S. Kennedy and M. H. Regier, "Prime Meridians In Medieval Islamic Astronomy, Vitas in Astronomy، vol. 28، No 1 (1985):29-32 accessed 1 March 2017,doi: 10.1016/0083-6656(85)90005-4.
أولغ بك (797 - 852 هـ 1) أحد

الفلكيين المسلمين المشهورين، وله الزيج تسمى بالزيج الجديد السلطاني، وهو خاتم لسلسلة المصنفات الفلاكية التي اقتفت أثر العلماء المأمونية وضمن فيه ولمديه 252 عدد أطوال المدن من الجزائر الخالدات. $2{ }^{2}$ أحد مبدأ الطول عند المتقمين. ${ }^{3}$ وعدد أطوال المدن المنات المدات يشير على فضله في تصنف الزيج فإن خطء عدد

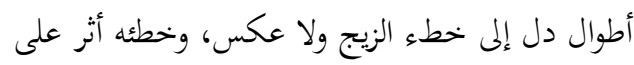

1اسمه عمد طور غاي بن شاه رخن بن تيمور كوركان

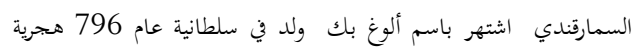

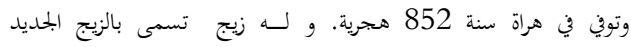

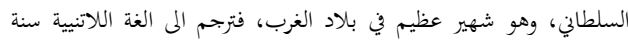

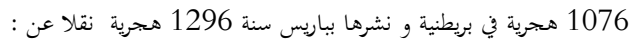

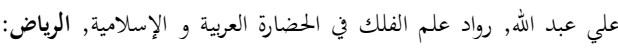

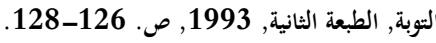

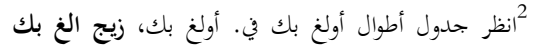

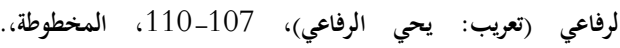
https://dl.wdl.org/3951/service/3951.pdf

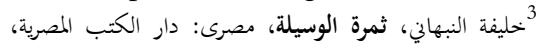

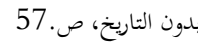


عنده، فطول بغداد عندنا يخالف بطوله، وكيفة تحديد الأطوال في عهده بالساعة المضبوطة من الأجرام السموية كالكواكب والقمر وبطريقة جيوديسية7. والإشارة لوقت متحد في عهد أولغ بك الساعة المضبوطة من الأجرام السموية كالكواكب والقمر, ولكن كسوفات قدمت من غيرها لأن غيرها لاتؤثر في حس البصر إلا في مدة مديدة لا يمكن فيها تمييز

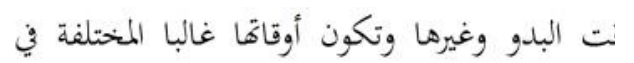

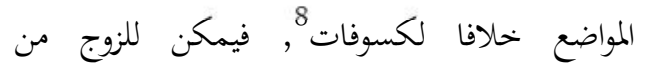
المراقبين، أحدها في كل منطقة، أن يراعي الأوقات

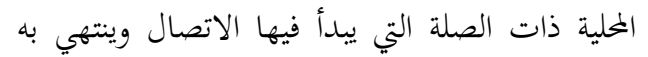
أقصى قدر من الانغماس أو الكلي. فنمكن أن نرسما هندسية تصحيح الأطوال باكسوفات على الرسم

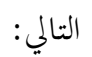

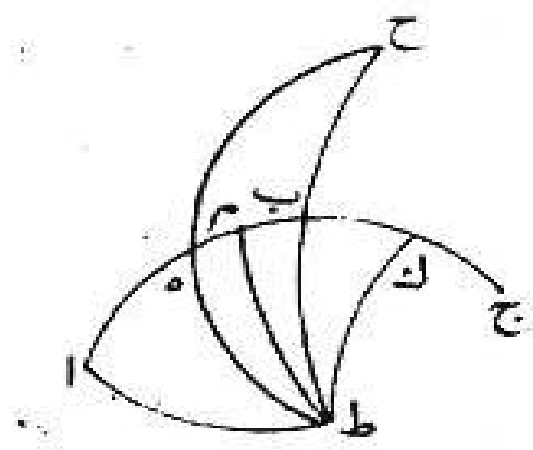

رسم 1: تصحيح الأطوال باكسوفات علي خط العرض الواحد. (المصدر: أبوالريحان البيروني, قانون المسعودي, ع ع الثاني الماني ص.510

7 Edward S. Kennedy, Mathematical Geography, P. 190, in Encylopedia of The History of Arabic Scince, Vol. I (Editet: Rashed, Roshidi and Morelon, Règis), (London: Routledge، 1996)

8 أبو الريحان البيروني، قانون المسعودي، ج. الثاني، الهند: دائرة المعارف العسمانية، 1955، ص.509.
الأخطاء في خط الطول عند العلماء المسلمين ليست

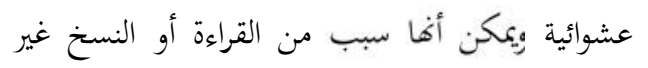
صحيحة بلا ملاحظة ومقارنة للبيانات الآخرى، فكانت هذه المقالة متممة موضحة للدراسة السابقة ب الناحية التي لم تبحث بها على التفصيل يعني خط مُه الطول عند أولغ بك خاصة. و بالإضافة إلى منهجية، تصنف هذه الرسالة في فئة البحث النوعية المكتبية (Research Library) باستفادة المنهج التاريخي (Historical Approach'). مصدر البيانات في هذه الدراسة إنقسم إلى مصدرين:

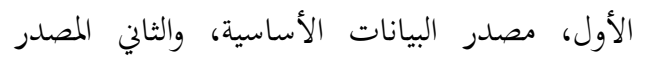
الثانوية. فالأساسية عبارة عن البيانات التي ترتبط مباشرة في المناقشة، واستفادت من الزيج الجديد

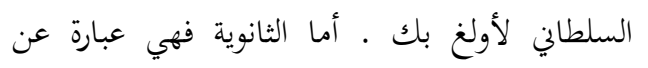
البيانات التي توضح المصدر الأساسية كمثل الوثائق والكتب وغيرها مما يرتبط موضوع البحث.

ب. فرضية شرح خطء أولغ بك في تحديد خط الطول حينما قرر مبدأ الطول كجرينتش فتحديد

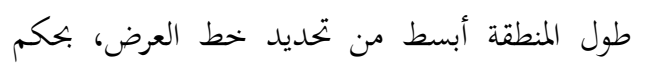

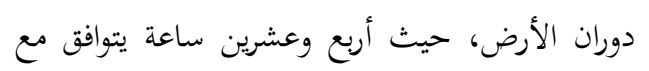

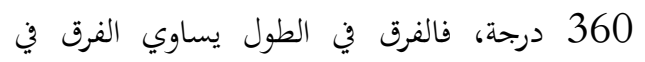
متوسط التوقيت المحلي بين المكانين. ولكن في الممارسة العملية هو الأصعب لندر إشارة الوقت المتحد في كل من المحليات ولعدم وجود وسائل الاتصال كراديو في عهد أولغ بك (797-852 هـ (

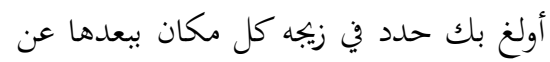

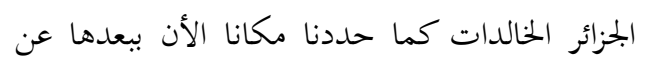
جرينتش، وعدد طول المدن عندنا يخالف بالطول

5 Nazir, Moh, Metode Penelitian، (Jakarta: Ghalia Indonesia، 1988) cet, III, hlm. 63

6 Suharsimi Arikunto، Prosedur Penelitian, (Jakarta: Rineka Cipta, 2013), 121. 
ه يشير إلى فضل الطولين، $\lambda$ هو خط

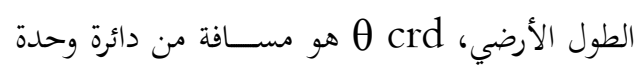

تحتها زاوية مركزية B, A,

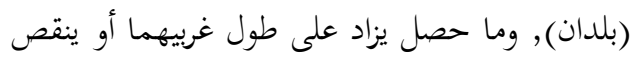

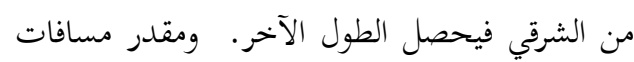

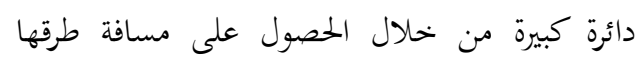

القافلة (فرسخ) ثم حولها إلى ميل ودرجات. فالذاتية

الفردية في طريقة جيوديسية هي أكبر من الطريق قبله,

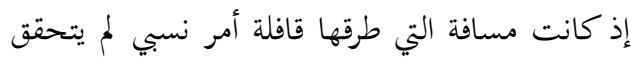

فيها, لاسيما مسافة التي أخبرها مسافر ـ وللطريقين في

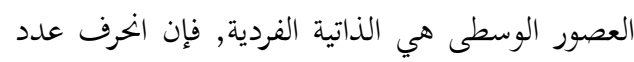

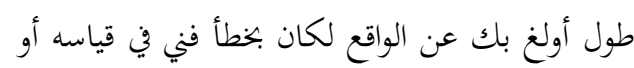

بأمر آخر، إذ كان الخطاء ليس بالقياس فقط ولم لوريكن

عدد الطول عندهم بالامتحان مضضا.

مقدر محيط الأرض في العصور الوسطى بلحئ

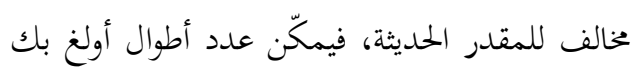

خخالف الواقع، لاختلافه البيانات الحديثية في كيفية

تحديد الأطوال و مقدر محيط الأرض، ولم تصل إلينا

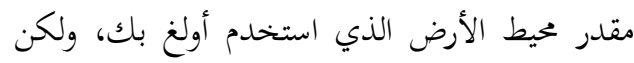
مقدر محيط الأرص عند علماء المسلمين مذهبان المأمونية و البيروني وهو 20400 ميل 9 و 20135 ميل 10، وكلهما حيث حوّل إلى كم 11 مخالفان المقدر الحديثة وإن كان قاربا بالواقع.

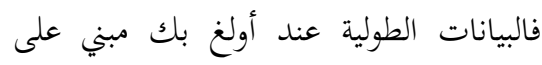

رصدية وجيوديسية خلافا لنا، فنستفيدها من القمر الصناعي وهو وافق الواقع لفقدان الذاتية الفردية. وأما

9 شاكر خصبك، الجغرافية عند العرب، بيروت: المؤسسة

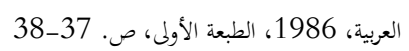

10

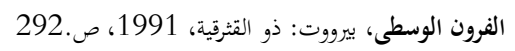

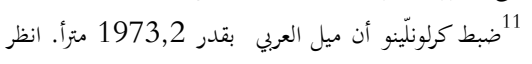

سنيور كرلنينّن، علم الفلك تاريخه عند العرب في الفرون الوسطى،
اب ج معدل النهار بين بلدين, ط ب ح حل

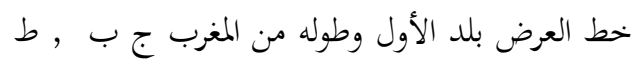

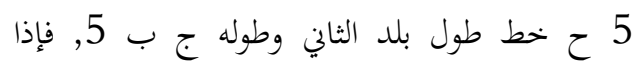

فرضنا كسوف إتفق في كلا بلدين غربيا على نقطة كل

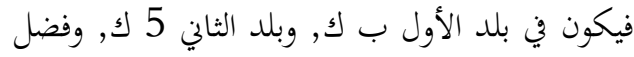

ما بينهما م الذي إذا زيد في طول ج بل بل حصل جل 5

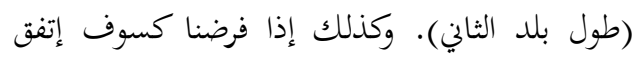

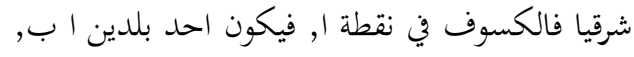

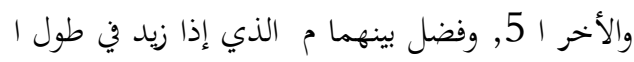
5 حصل ا ب (طول بلد الأول).

أما طريقة جيوديسية فتصحيح طول البلدان بولن

بالمسافات. فنفترض أن خطوط العرض في بلدان معروفة ( A و C في الرسم الثاني), وكذا مسافة التي

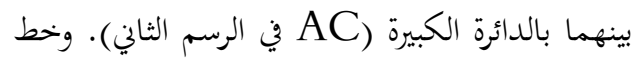
الطول وموازية من خط العرض يمر بنقطتين سابقين, وتتقاطع هذه الدوائر الأربع في أربع نقاط التي بأجزاء مستقيمة على شبه منحرف.

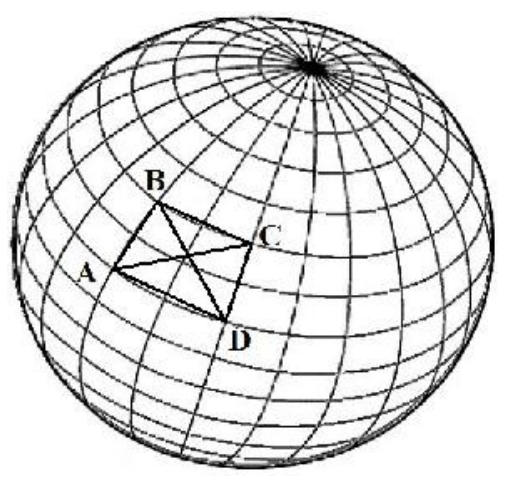
رسم 2: شبه منحرف متساوي الساقين لمكانين C , A على الأرض. (ألمصدر: جوجل)

ظه =CD , AC = فهر في ذلك الرسم أن فالمعلوم $A C^{2}=. \mathrm{AB}^{2}+\mathrm{BC} \cdot \mathrm{AD}$ ف في ذلر ان أن AC مسافة و فضل خطي العرض بين المكانين, فوجدنا منها جيب فضل ما بين طولي المكانين باستخدام وظيفة المثلثية، وكان صيغته ما يلي: $\Delta \lambda=\operatorname{arc} \operatorname{crd} \sqrt{\left[\frac{\operatorname{crd}^{2}(\widehat{\mathrm{AB}})-\operatorname{crd}^{2}(\Delta \phi)}{\cos \phi_{\mathrm{A}} \cos \phi_{\mathrm{B}}}\right]}$ 


$$
\begin{aligned}
& \text { بياناته فلا تزال في الذاتية الفردية، فتباين البيانات عكسه. أبسط فج هو أن نفترض أن هذا التحويل }
\end{aligned}
$$

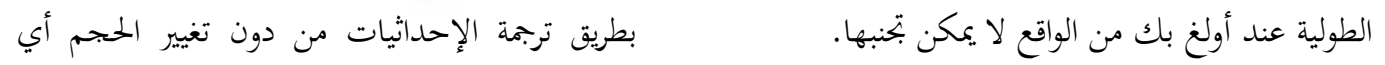

$$
\begin{aligned}
& \text { ج. مقارنة خطوط الطول أولغ بك مع خطوط الطع لا يكن لخنها. }
\end{aligned}
$$

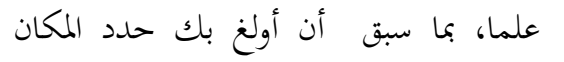

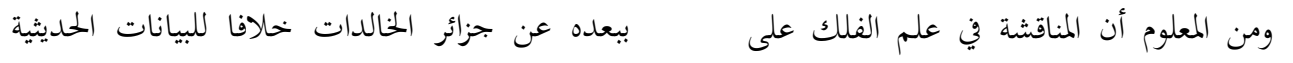

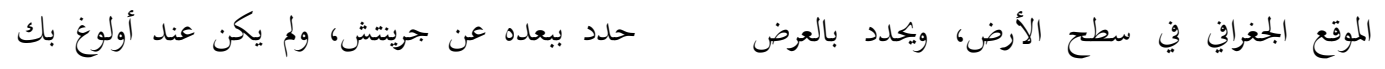

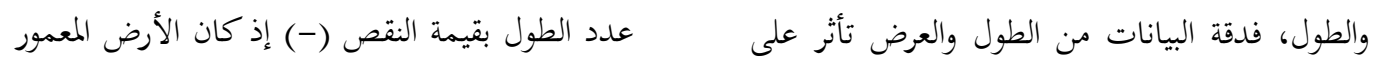

$$
\begin{aligned}
& \text { عنده يسع } 180 \text { جه طولا، فكالحصير المقوس وجزائر } \\
& \text { الخالدات وقع في غربي جرينتش فاقتضى به أن مناطق }
\end{aligned}
$$

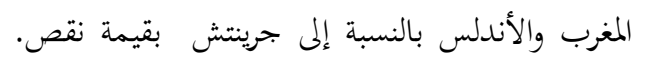

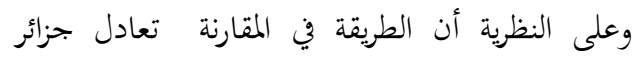

$$
\begin{aligned}
& \text { الخالدات بجرينتش في القيمة الثابتة، وكانت المعادلة } \\
& \text { بطرح عدد طول المدن من جرينتش بعدد طول المدن }
\end{aligned}
$$

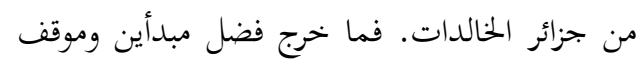

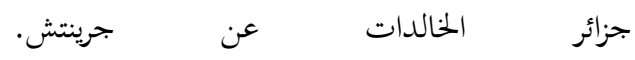

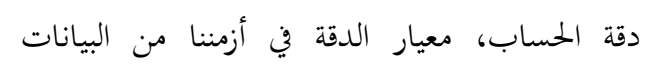

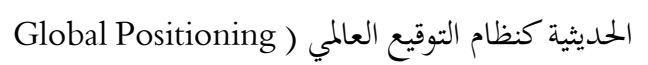

$$
\begin{aligned}
& \text { System وجوجل إيرث. وفي تحليل مقارنة أطوال } \\
& \text { البلدان عند أولغ بك مع أطوال البلدان الحلديثية }
\end{aligned}
$$

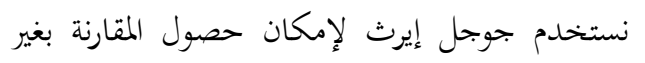

$$
\begin{aligned}
& \text { حضور على الموقع المعينة. }
\end{aligned}
$$

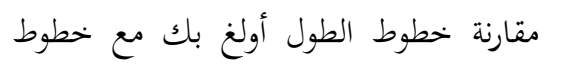

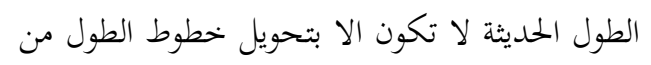

$$
\begin{aligned}
& \text { جزائر الخالدات إلى خطوط الطول من جريتش، وكذا }
\end{aligned}
$$

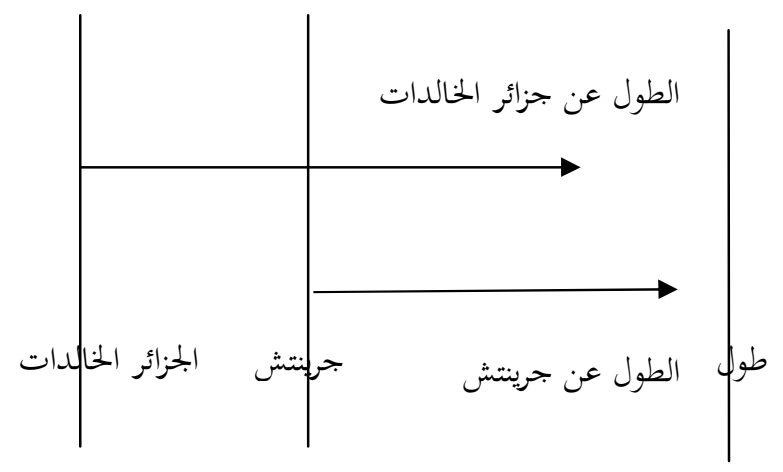

$$
\begin{aligned}
& \text { حتى عصرنا. } \\
& \text { جريتتشية. }
\end{aligned}
$$


والواقع لم يكن في فضل ثابت بين جزائر الخالدات

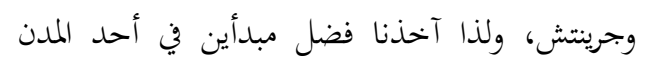
ثابتا يتمثل كل طول البلدان. فاخترنا أحد طول المدن آندان ألمان مرجيعية (بع جك) لغيره، ثم نعادله إلى خط الطول

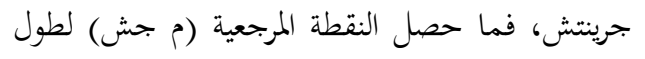

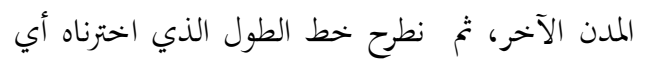

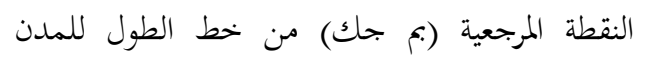
الأخر (م جك)، فحصل فضل بين طولين، ثم النقطة النقال

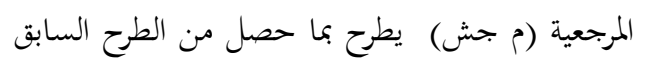

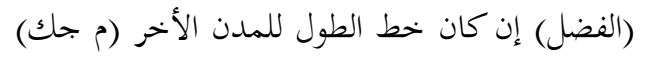
أقصر من طول المدن مرجيعية (بع جك) ونزيده إن إن الن المان كان أكبر فما خرج عدد التحويل المطلوب ،لإيضاح صيغة التي كانت الماضية أن نرمهها على النحو التالي:

\begin{tabular}{|c|c|c|c|}
\hline \multirow{2}{*}{ فبدأين } & \multicolumn{2}{|c|}{ طول البلدان } & \multirow[b]{2}{*}{ أسمآء المددن } \\
\hline & جرينتش & الخالدات & \\
\hline Tr,Tr & ro,or & $7,1 \mu$ & طبرية \\
\hline rT,qR & $r_{0, r}$. & 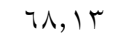 & صور \\
\hline$\mu_{r}, r_{\Lambda}$ & $r_{0, T}$. & $7 \Lambda, 0 \wedge$ & صردا \\
\hline$r r, \Lambda r$ & $\Gamma_{0, \Lambda \Gamma}$ & $79,7 \mathrm{~V}$ & طرابلس لبناءن \\
\hline rr,Ar & $r q, r v$ & $v \cdot, \cdot \wedge$ & دمشق \\
\hline$r \varepsilon, \infty 0$ & $r q, r$. & $v \cdot, v_{0}$ & بعلبك \\
\hline$r \varepsilon, 0$ & $r q, v$. & $\vee \cdot, v_{0}$ & حمص \\
\hline$r_{0, r \wedge}$ & $r, 10$ & $v 1, \Sigma r$ & أنطاكيا \\
\hline$r \varepsilon, \varepsilon$. & $r \tau, v T$ & $v 1,1 r$ & سرمين \\
\hline$r_{0,1}$ & $\cdots, r V$ & $V T, I r$ & قنسرين \\
\hline
\end{tabular}

اتضح من الجدول السابق أن في عدد الطول عند أولغ بك لم يكن له فضل ثابت بأطوال البلدان

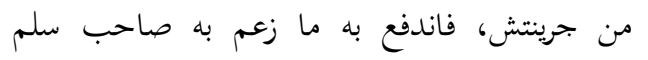

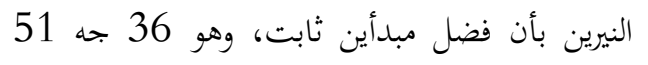

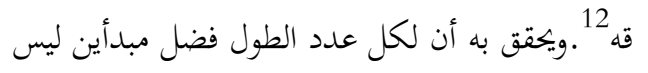

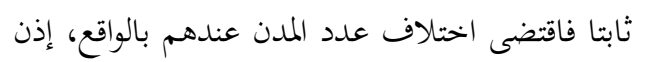

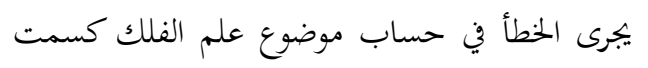
القبلة وأوقات الصلوة وغيرها. فاستحال علينا تحويل عدد الطول من جزائر الطرات الخالدات إلى الطول الجريتشية الا باختيار النقطة

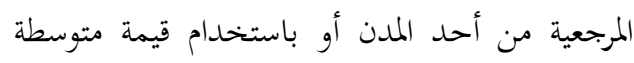

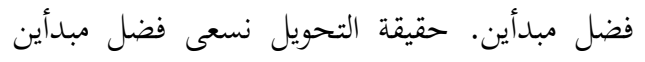

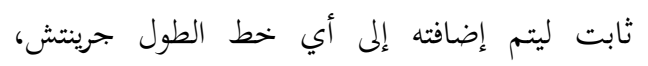

12 1ممد منصور، سلم النيرين، كادري: الفلاح فالوصا، بدون التاريخ، الجدول التاسع عشر. 


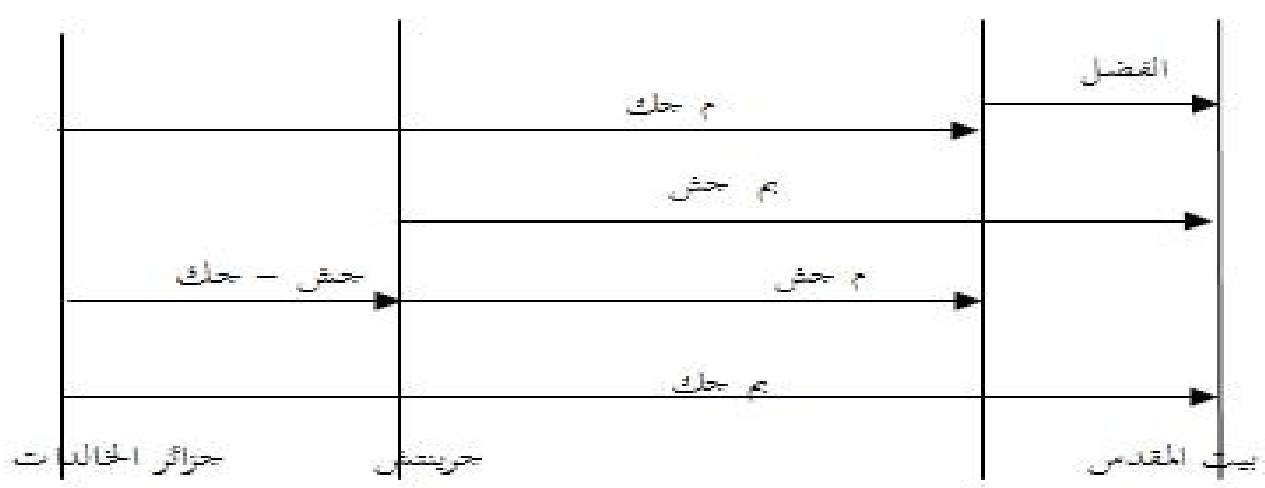

رسم 4: التحويل طول من جزائر الخالدات إلى طول جريتشية.

العرض لكل المدن، ففرضنا أن عدد العرض عندهم

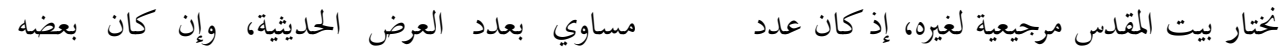

طوله مستويا عند علماء المسلمين في العصور مختلف. أما حاصل التحويل لأطوال مذكورة فوضعنا

الوسطى، فيمكن عدد طوله بالممتحن. كل عدد طول ي الجدول سيأتي، و رسمنا الحاصل في خريطة. وهو ما

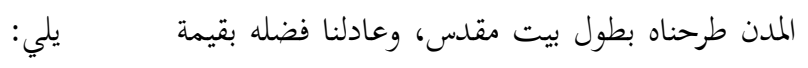
جرينتش كما سبق. ولم نلاحظ في معادلته عدد

\begin{tabular}{|c|c|c|c|c|c|}
\hline \multirow[b]{2}{*}{ الخطأ } & \multirow{2}{*}{ التحويل } & \multirow{2}{*}{ فضدأين } & \multicolumn{2}{|c|}{ طول البلدان } & \multirow[b]{2}{*}{ أسمآء المدن } \\
\hline & & & جرينتش & الخالئر & \\
\hline سז, 1. & 10, & rr, & ro,or & $\uparrow \wedge, 1 \Gamma$ & طبرية \\
\hline $.1,70$ & $r \uparrow, \wedge 0$ & rr,qr & $r_{0}, r$. & $\neg \wedge, 1 r$ & صور \\
\hline$\cdot r, 1 \cdot$ & $r v, r$. & גז, & $r_{0, r}$. & $\top \wedge, \diamond \wedge$ & صردا \\
\hline$\cdot r, 00$ & 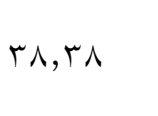 & س & ro, & $79,7 V$ & طرابلس \\
\hline$\cdot r, O r$ & $\longleftrightarrow \wedge, \wedge$. & r, & $r \Psi, r V$ & $v \cdot, \cdot \Lambda$ & دمشق \\
\hline$\cdot r, r V$ & $r q, \Sigma v$ & $\Gamma \varepsilon, 00$ & $r q, r$. & $v \cdot, v_{0}$ & بعلبك \\
\hline$\cdot r, V V$ & $r q, \leqslant V$ & $\Gamma \varepsilon, 0$ & $r q, v$. & $v \cdot, v_{0}$ & حصص \\
\hline$\cdot \varepsilon, \cdots$ & $\varepsilon \cdot, 10$ & ro, rᄉ & $r 7,10$ & $v 1, \Sigma r$ & أنطاكيا \\
\hline$\cdot r, 1 r$ & $r q, \wedge r$ & $r \varepsilon, \varepsilon$. & $r u, v r$ & VI, Ir & سرمين \\
\hline$\cdot r, \wedge 0$ & $\varepsilon \cdot, \wedge 0$ & ro, & rv,.. & VY,Ir & قنسرين \\
\hline
\end{tabular}

جدول 2: تحويل إقليم الشام لأولغ بك إلى الخط جرينتشية. 
الخطعء فيه عوامل محتملة مخطئ في المقدار للمسافات والتحويل الخاطئ لقياس المسافة إلى الدرجات. ويتحقق واحدا منها،وهو الخطء في قياس محيط

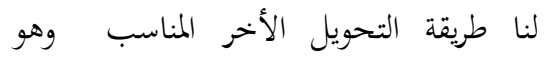

باستخدام قيمة متوسطة فضلا ثابتا. ونخن في حاجة

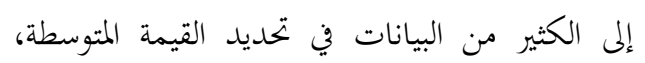
بحيث متوسط فضل مبدأين والخطإ العشوائي أدقة

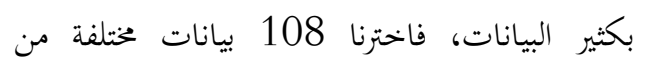
252 بيانات عند أولغ بك. فقدر القيمة المتوسطة عند أولغ بك 33 جه 42 قه 59 ذي. فالمثير

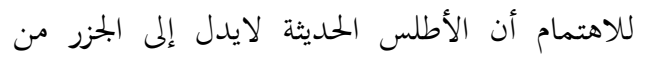

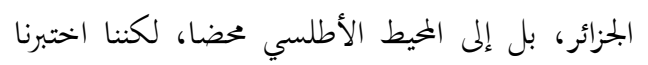

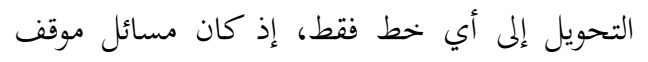

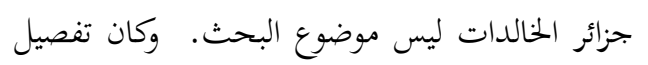

$$
\begin{aligned}
& \text { التحويل فيما يلي: }
\end{aligned}
$$

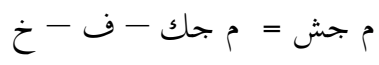

$$
\begin{aligned}
& \text { م جك = م جش + }
\end{aligned}
$$

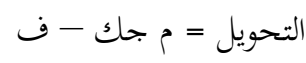

$$
\begin{aligned}
& \text { معلومة الصيغة : }
\end{aligned}
$$

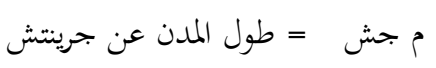

$$
\begin{aligned}
& \text { م جك = طول المدن عن جزائر الخالدات }
\end{aligned}
$$

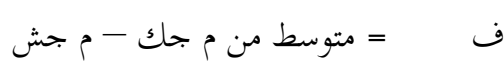

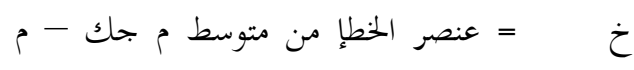

$$
\begin{aligned}
& \text { جش. }
\end{aligned}
$$

استنادا إلى الصيغة في الأعلا، يجب علينا

مقدما بالضرورة الاكتشاف على القيمة متوسطة من كافة البيانات كلها، ويمكننا بها أن نحلل كمية الخطأ من أطوال المدن عند أولغ بك، فاختبرنا بيانات الأطوال الممكنات تحديدها في عصرنا ولكننا نقيم

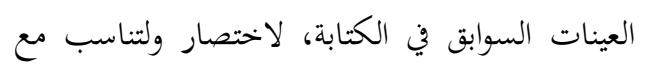

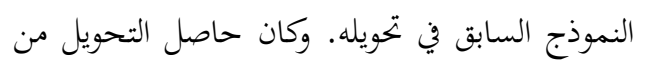
العينات نرسمه في الجدول وفي خريطة. وهو ما يلي:
اتضح من العيّة الماضية في الجحدول أن أطوال

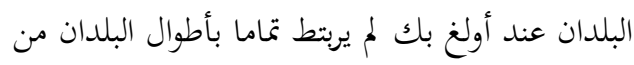

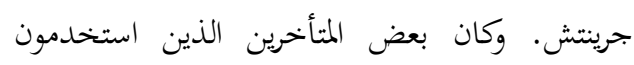
جزائر الخالدات مبدأ الطول كصاحب سلم النيرين زعم أن فضل مبدأين ثابت. فإذا سلمنا معلومته لكان

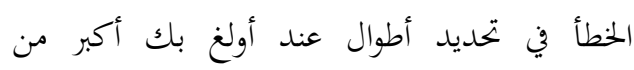
التحويل السابق في منطقة الشام كدمشق للفضل

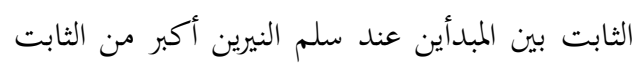
الذي أخذناه من النقطة المرجعية التي تقدم. وأما المنطقة الأخرى فيقتضي كذلك الا أن فضل مبدأين الخدين

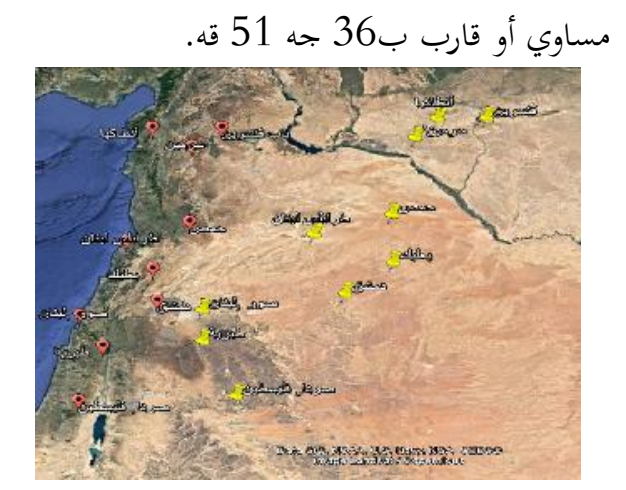

$$
\begin{aligned}
& \text { = طول المدن أولغ بك وكان مستنبطا من نقطة } \\
& \text { بيت المقدس }
\end{aligned}
$$$$
\text { 央 }
$$

رسم 5: مقارنة خط الطول من جزائر الخالدات عند أولغ بك مع خط الطول من جرينتش في إقليم الشام.

$$
\text { النقطة المرجعية هو بيت المقدس. }
$$

دل الرسم إلى أن كل عدد طول المدن عند أولغ بك في منطقة الشام وقع في شرقي من الحقائق

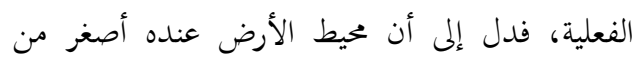

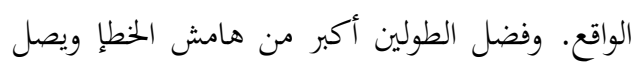

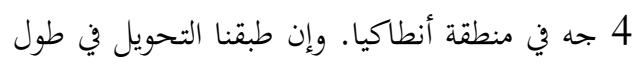

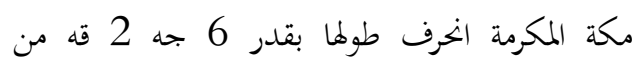
الحقائق الفعلية، فالفضل الذي حدث في أطوال أولغ

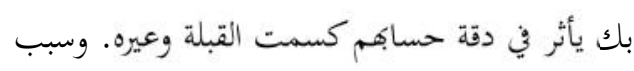




\begin{tabular}{|c|c|c|c|c|c|}
\hline \multirow[b]{2}{*}{ التحويل } & \multirow{2}{*}{$\begin{array}{c}\text { العشوائي } \\
\text { الخطإ) } \\
\text { إخ }\end{array}$} & \multirow{2}{*}{ فبدأين } & \multicolumn{2}{|c|}{ طول البلدان } & \multirow[b]{2}{*}{ أسمآء المدن } \\
\hline & & & جرينتش & جزائر & \\
\hline$r \varepsilon, \varepsilon r$ & $-1,1$. & rT,Tr & $r_{0,0 T}$ & $7 \wedge, 1 r$ & طبرية \\
\hline$r \varepsilon, \varepsilon r$ & $-\cdot, \vee \wedge$ & rT,qT & $r_{0, r}$. & $\uparrow, 1 r$ & صور \\
\hline$\wedge \vee, r \varepsilon$ & $-\cdot, r \mu$ & ט r & $r_{0, r}$. & $7 \wedge, 0 \wedge$ & صردا \\
\hline$r_{0,90}$ & $\cdot, 1 T$ & rr,人 & $r_{0, \Lambda}$ & $79,7 V$ & طرابلس لبنان \\
\hline$r q, r v$ & $\cdot, 1$. & rT, & T T, rV & $v \cdot, \cdot \wedge$ & دمشق \\
\hline$r v, \cdot r$ & $\cdot, \wedge r$ & $r \varepsilon, 00$ & $r ד, r$. & $v \cdot, v_{0}$ & بعلبك \\
\hline$r v, \cdot r$ & תr, & $r \varepsilon, .0$ & $r u, v$. & $v \cdot, v_{0}$ & حمص \\
\hline$r V, V T$ & $1,0 \mathrm{~V}$ & $r_{0, r \Lambda}$ & $r 4,10$ & $v 1, \varepsilon r$ & أنطاكيا \\
\hline$r v, \varepsilon$. & $\cdot, \uparrow$ & $r \varepsilon, \varepsilon$. & $r T, V T$ & $V 1,1 r$ & سرمين \\
\hline rᄉ, $\leqslant r$ & $1, \varepsilon r$ & $r_{0,1}$ & $r v, \ldots$ & VT,Ir & قنسرين \\
\hline
\end{tabular}

جدول 2: تحويل إقليم الشام لأولغ بك إلى الخط جريتشية.

رسم 6: مقارنة خط الطول من جزائر الخالدات عند أولغ بك مع خط الطول من جرينتش في إقليم الشام. وكان مستنبطا من القيمة المتوسطة في فضل المبدأين اتضح من الخريطة السابقة أن تحويل طول المدن إلي في لمئل خط طول جرينتش بالقيمة المتوسطة من فضل المبدأين

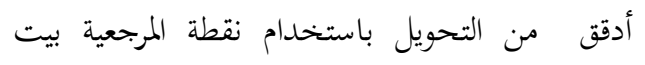

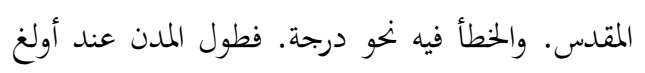

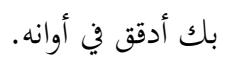

الارتباط والمقارنة بين البيانات أولغ بك

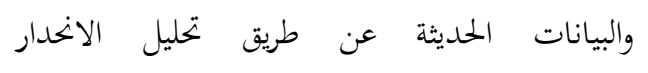
فبتحليلها موضح البيانات (regression analysis) الطولية المردودة ومصور معادلة جديدة لتحويل البيانات الطولية أولغ بك إلى البيانات الحديثة،

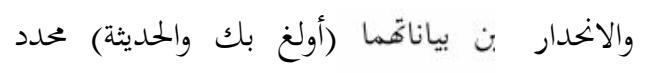

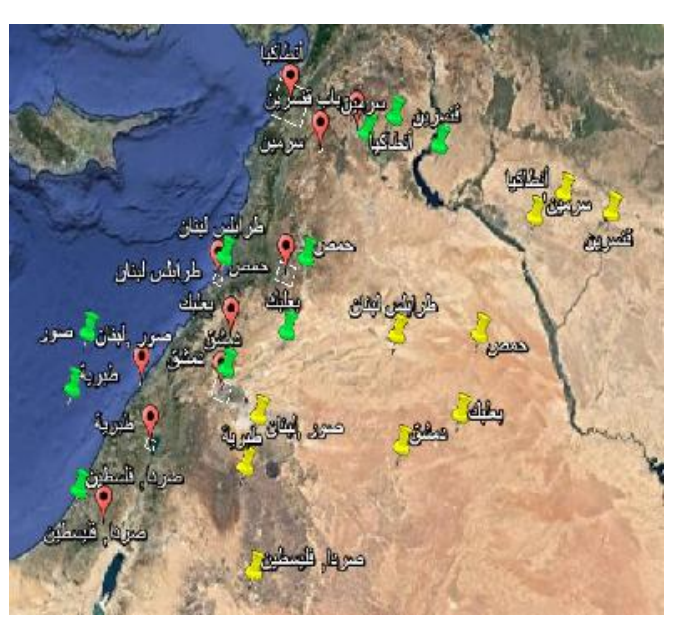
بالمعادلات الخطية. إذا حللنا مقارنة بين بيانات = طول المدن بالفعلي (الواقع) ק = طول المدن عند أولغ بك وكان مستنبطا من نقطة ست المقدس

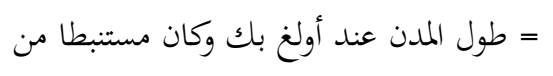
القيمة المتوسطة

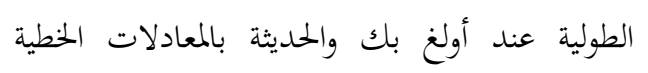


كان أدقة في أوانه، وأن الارتباط طول أولغ بك مع طول الحديثة لم يرتبط تماما. وبناء على المعادلة التي كانت السابقة يمكننا وضع عدد طول المدن عند أولغ

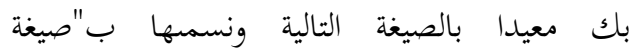
الانحدار". إعادة حساب الطول = (طول أولغ بك - بكابك

$$
.1,063 /(31,23
$$

معظم عدد الأطوال في زيج أولغ بك تختلف

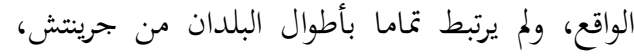

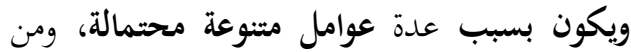
عوامل محتملة غخطئ في المقدار للمسافات والتحويل الخاطئ لقياس المسافة إلى الدرجات، ويتحقق واحدا منها، وهو الخطء في قياس محيط الأرض. نتائج البحث لدينا يمكن تفصيلها وتصحيحها والمنازع عليها, والمقصود من هذا البحث هو دعوة لمزيد من المناقشة خط الطول في العصور الوسطى. وكان نتائجنا لا تدحض حال الفرضية التي كانت بمثابة نقطة

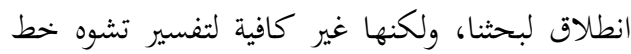

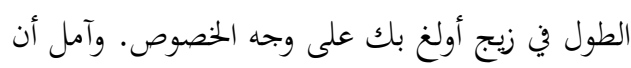

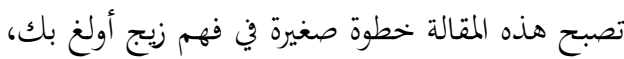

$$
\text { حيث خط طوله. }
$$

حصل لنا الانحدار والإتباط r) بينهما، فإن كان

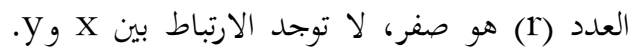

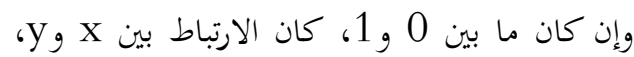

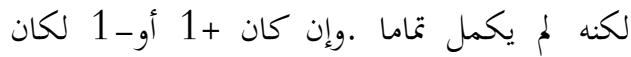
المتغيران مترابطة.

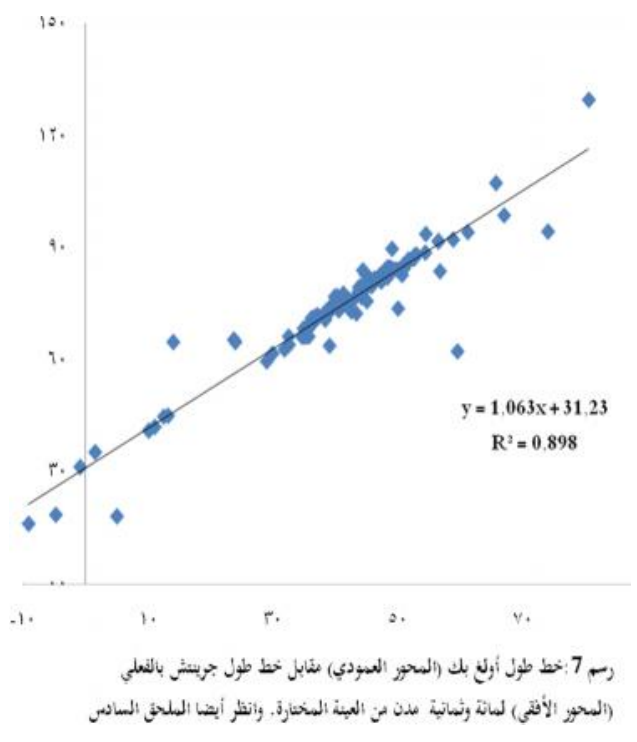

دلت التصميمات إلى أن البيانات الطولية عند

أولغ بك لايناسب ولا يقارب الواقع الا منطقة عراق

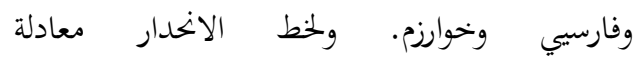

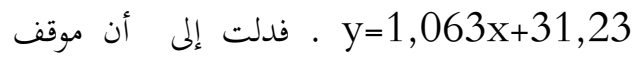
مبدإ الطول عند أولغ بك عن غربي جرينتش بقدر

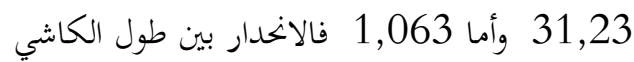
والطول الجرينتشية. وقيمة R2 بعيدة من 1 فتظهر بوضوح أن عدد الطول عند أولغ بك بطريقة خطية 


\begin{tabular}{|c|c|c|c|c|c|c|}
\hline \multicolumn{3}{|c|}{ التحويل } & \multirow[b]{2}{*}{ 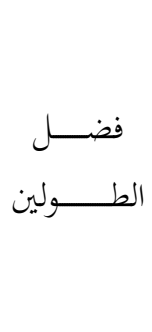 } & \multicolumn{2}{|c|}{ طول البلدان } & \\
\hline صيغة & متوسطة & مرجعية & & جرينتش & الخالداتر & أسمآء المدن \\
\hline$\cdot \varepsilon, \cdot r$ & $\cdot 1, \vee \wedge$ & $\cdot \varepsilon, r T$ & $r 7,9 r$ & $\cdot \wedge, \circ \wedge$ & ro,o. & السوس الأقصى \\
\hline$-I r, r r$ & $-10,01$ & $-1 T, 10$ & & 0 & IN, & فاس \\
\hline$\cdot \varepsilon, \cdot r$ & $\cdot 1, \vee \wedge$ & $\cdot \varepsilon, Y T$ & rr,9人 & $.1,0 Y$ & ro,o. & تاهرت \\
\hline$\cdot\urcorner, \vee \wedge$ & $\cdot \varepsilon, V T$ & $\cdot v, 10$ & ס T, & $-\varepsilon, \vee \wedge$ & rᄉ, $\Sigma$ & قرطبة \\
\hline $.9, T V$ & $\cdot V, r V$ & $\cdot 9, \wedge$. & r & $\cdot \wedge, 1 \cdot$ & $\leqslant 1,1$ & القيروان \\
\hline $1 \cdot, r_{7}$ & $\cdot \Lambda, \leqslant Y$ & $1 \cdot, 10$ & r & 11,0 & $\varepsilon r, 1 T$ & مهدية \\
\hline $1 T, \lambda$ & $11, \leqslant r$ & $1 r, \wedge 0$ & r1,90 & $|r,| \wedge$ & $\varepsilon 0,1 \mu$ & طرابلس المغرب \\
\hline$r \wedge, \wedge \circ$ & 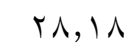 & $r \cdot, T r$ & rI,91 & $r q, q r$ & $71,9$. & إسكندرية \\
\hline ro, 70 & $r \varepsilon, V \wedge$ & $r V, r T$ & $r\urcorner, \uparrow \wedge$ & r,人r & $\circ \wedge, 0$. & دمياط \\
\hline$r \cdot, 90$ & $r \cdot, \leqslant r$ & $r$ r, & r & rr,oo & $7 \varepsilon, 1 T$ & قلزم (السويس) \\
\hline$r q, \varepsilon$. & $\varepsilon \cdot, \leqslant 0$ & $\varepsilon r, \wedge \wedge$ & $r \cdot, \wedge \circ$ & $\varepsilon r, r r$ & $v \varepsilon, 1 V$ & زبيد \\
\hline$\leqslant 1, \leqslant 9$ & $\{1,7\}$ & $\varepsilon \varepsilon, \cdot 0$ & $r_{0}, V V$ & $r q, \circ v$ & ru,r & مدينة المنورة \\
\hline$\varepsilon T, T \leqslant$ & $\varepsilon r, \varepsilon r$ & $\varepsilon \varepsilon, \wedge 0$ & r & $\varepsilon 0, \ldots$ & V7, r & عدن \\
\hline$\varepsilon r, 1 \wedge$ & $\varepsilon r, \varepsilon r$ & $\leqslant 0, \wedge 0$ & rr, 90 & $\varepsilon \varepsilon, 1 \wedge$ & $V V, I r$ & صنعاء \\
\hline$\varepsilon r, r)$ & $\varepsilon r, \leqslant 0$ & $\varepsilon 0, \wedge \wedge$ & $r V, r r$ & $r q, \wedge 0$ & $V V, I V$ & مكة الكرمة \\
\hline$\varepsilon r, O r$ & $\varepsilon r, \vee \wedge$ & $\varepsilon 7, r\}$ & $r v, \ldots$ & $\varepsilon \cdot, 0$. & $V V, 0$. & طائف \\
\hline$\varepsilon q, \vee \vee$ & $0 ., \leqslant Y$ & or,, 10 & $r V, \Sigma r$ & $07, V$. & $\Lambda \varepsilon, 1 \Gamma$ & صحار \\
\hline 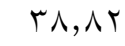 & 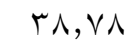 & $\varepsilon 1, r\}$ & $r 0, r$. & $\varepsilon V, r$. & VT,O. & يمامة \\
\hline$\varepsilon \cdot, \Gamma_{\tau}$ & $\varepsilon \cdot, \varepsilon r$ & $\leqslant r, \wedge 0$ & $r \varepsilon, 1 r$ & $0 \cdot, \cdot$ & $v \varepsilon, 1 r$ & قطيف \\
\hline rч, & $r \uparrow, \leqslant r$ & r人, ^o & $19,0$. & $0 \cdot, 7$ & $v \cdot, 1 r$ & هجر \\
\hline & rr, VA & ro,rr & r, ז人 & ro,rr & $77,0$. & بيت المقدس \\
\hline 人 & r, VA & ro,r & r, r & $r \varepsilon, O V$ & $77,0$. & عسقلان فلسطين \\
\hline 人 & r, VN & ro,rr & $r \cdot, \Lambda T$ & ro, T人 & $77,0$. & قيسارية \\
\hline$r r, \varepsilon q$ & rr, & ro, 00 & ri, 9V & $r \varepsilon, \wedge V$ & $\neg 7, \wedge r$ & رملة فلسطين \\
\hline$r \varepsilon, V T$ & $r \varepsilon, \varepsilon r$ & r,人o & rr, & ro,or & T人, Ir & طبرية \\
\hline
\end{tabular}




\begin{tabular}{|c|c|c|c|c|c|c|}
\hline ro, $T$ & $r \varepsilon, \vee \wedge$ & rV, r & . & $r_{0, r}$. & $71,0$. & صور \\
\hline$r_{0}, 1 \varepsilon$ & $r \varepsilon, \wedge \vee$ & $r \vee, r$. & 人ץ, & $r_{0, r}$. & $7 \wedge, 0 \wedge$ & صردا \\
\hline Tr,1 & ro, 90 & 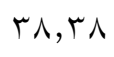 & س & س & $79,7 V$ & طرابلس لبنان \\
\hline r & vז, & 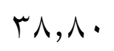 & r, & $r \tau, r V$ & $v \cdot, \cdot \Lambda$ & دمشق \\
\hline$r v, 1 \wedge$ & $r v, \cdot r$ & $r q, \leqslant V$ & $r \leqslant, 00$ & $r \tau, r$. & $V \cdot, v_{0}$ & بعلبك \\
\hline$r \vee, 1 \wedge$ & rv, r & $r q, \leqslant V$ & $r \varepsilon, 0$ & r & $v \cdot, v_{0}$ & ص \\
\hline$r V, \wedge r$ & $r V, V T$ & $\varepsilon \cdot, 10$ & ro, rᄉ & r & VI, $\leqslant r$ & أنطاكيا \\
\hline rV,Or & $r v, \varepsilon$. & $r q, \wedge r$ & $r \varepsilon, \varepsilon$. & $r \tau, V T$ & $V 1,1 r$ & سرمين \\
\hline 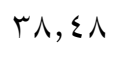 & $\varepsilon r, r \wedge$ & $\varepsilon \cdot, 10$ & r & rv,.. & VT, Ir & قنسرين \\
\hline$r \wedge, 01$ & $r \wedge, \Sigma 0$ & $\varepsilon \cdot, \wedge \wedge$ & $r_{0}, r$ & rV, Ir & $V T, I V$ & حلب \\
\hline ro,rv & ro, & $r v, 00$ & ه , & $r \varepsilon, \wedge \wedge$ & $\neg \Lambda, \Lambda r$ & طرسوس \\
\hline $\mid V, 01$ & $17, r$. & מוֹ & $1 \varepsilon, r$. & ro, r & $\varepsilon q, 9\}$ & مصيصة \\
\hline$r v, 0 \leqslant$ & $r v, \varepsilon r$ & $r q, \wedge 0$ & $r r, \Lambda$. & r r & $v 1,1 r$ & ملطية \\
\hline r,$\wedge q$ & $r, \leqslant r$ & & 01,11 & $1 \varepsilon, \cdot r$ & 70,11 & صقيلية \\
\hline$\cdot r, q \vee$ & $.1, V \mu$ & $\cdot \varepsilon, 1 V$ & $r r, 90$ & $1 T, 0$. & ro, $\leqslant 0$ & رمية كبرى \\
\hline$r 7,91$ & $r 7,1 r$ & rᄉ,, 0 & $r \cdot, \wedge V$ & r^, $9 \vee$ & $\circ q, \wedge r$ & قسطنطنية \\
\hline$r$ r, $\Sigma$. & 1,90 & r & $\leqslant 1,90$ & $r r, V r$ & $70,7 \mathrm{~V}$ & أثينة \\
\hline$r V, 19$ & $r\urcorner, \Sigma r$ & rᄉ, $\wedge 0$ & ג & rT,90 & & ماقندونيا \\
\hline$r \cdot, 90$ & $r \cdot, \Sigma r$ & r,人o & ro, Ir & $r q, \cdot r$ & $T \varepsilon, 1 T$ & عمورية \\
\hline$\varepsilon r, 1 \wedge$ & $\varepsilon r, \varepsilon r$ & $\leqslant 0, \wedge 0$ & 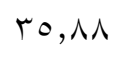 & $\leqslant 1, r_{0}$ & $V V, I r$ & إرزالروم (أرضروم) \\
\hline rᄉ, $\cdot \varepsilon$ & rv, 90 & $\varepsilon \cdot, \Lambda$ & $r \varepsilon, 7 V$ & $r v, \ldots$ & $V I, T V$ & بواس \\
\hline rᄉ, q. & ऍ, $\wedge \vee$ & $\varepsilon 1, \Gamma$. & $r \varepsilon, 1 \Gamma$ & ऍ & $V Y, O \wedge$ & سميساط \\
\hline$\varepsilon \varepsilon, r q$ & $\varepsilon \varepsilon, V$. & $\varepsilon \vee, I r$ & $r_{0}, q r$ & $\varepsilon r, \Sigma \wedge$ & $V \wedge, \leqslant Y$ & قاليقلا \\
\hline & rr, VA & ro, r & $r \varepsilon, \cdot r$ & $r\}, \Sigma \Lambda$ & $77,0$. & قوينة \\
\hline$\varepsilon \varepsilon, 1\}$ & $\varepsilon \varepsilon, \varepsilon r$ & $\leqslant 7, \wedge 0$ & $r q, 1 r$ & $r q, \cdot r$ & $\vee \wedge, I r$ & حران \\
\hline$\varepsilon \varepsilon, \wedge$ & $\varepsilon \varepsilon, r v$ & $\varepsilon \neg, \wedge$. & $r q, \cdot \wedge$ & $r q, \ldots$ & $\vee \wedge, \cdot \wedge$ & الرقة \\
\hline$\varepsilon \cdot, r \tau$ & $\varepsilon \cdot, \varepsilon r$ & $\varepsilon r, \wedge 0$ & $r \varepsilon, \cdot V$ & $\varepsilon \cdot, \cdot v$ & $v \varepsilon, 1 r$ & رأس العين \\
\hline$\varepsilon \cdot, r q$ & $\varepsilon \cdot, \varepsilon r$ & $\varepsilon r, \wedge 0$ & r & $\varepsilon \cdot, \vee \mu$ & $v \varepsilon, 1 T$ & ماردين \\
\hline$\varepsilon \cdot, \wedge\rceil$ & $\varepsilon \cdot, 90$ & $\varepsilon r, r \wedge$ & $r \varepsilon, r_{0}$ & $\varepsilon \cdot, \Sigma T$ & $V \varepsilon, T V$ & قرقيشيا \\
\hline$\leqslant 1, \leqslant 9$ & $\Sigma 1,7 r$ & $\varepsilon \varepsilon, 0$ & $r \varepsilon, 1 r$ & $\varepsilon 1, r$. & س & نصيبين \\
\hline
\end{tabular}




\begin{tabular}{|c|c|c|c|c|c|c|}
\hline$\leqslant r, 09$ & $\varepsilon r, \vee \wedge$ & $\varepsilon 0, r\}$ & $r \varepsilon, O r$ & $\leqslant 1,9 \wedge$ & $V 7,0$. & عانة \\
\hline$\varepsilon r, \mid \wedge$ & $\Sigma T, \Sigma T$ & $\varepsilon 0, \wedge 0$ & ג, & $\varepsilon r, 10$ & $V V, I r$ & \\
\hline$\varepsilon r, r V$ & $\varepsilon r, T r$ & $\leqslant 7,0$ & אז, זr & $\varepsilon \leqslant, \cdots$ & سr, & أربيل \\
\hline$\varepsilon \varepsilon, \Gamma$ & $\varepsilon \varepsilon, T Y$ & $\varepsilon \vee, .0$ & $r \varepsilon,\rceil \wedge$ & $\varepsilon r, 70$ & VA,r & تكريت \\
\hline$\varepsilon \varepsilon, 7 T$ & $\varepsilon \varepsilon, 90$ & $\varepsilon \vee, r \wedge$ & rᄉ, $\Sigma V$ & $\varepsilon \cdot, r$. & $\vee \wedge, T \vee$ & آمد (ديار بكر) \\
\hline$\{1,97$ & $\varepsilon r, I r$ & $\varepsilon \leqslant, \infty 0$ & סr, rr & $\varepsilon r, \varepsilon \wedge$ & Vo, & أخلاط \\
\hline$\varepsilon 0, \cdot 7$ & $\varepsilon 0, \Sigma T$ & $\varepsilon \vee, \wedge 0$ & $r_{0}, \Sigma r$ & $\varepsilon r, v$. & $\vee q, 1 r$ & أنب \\
\hline$\cdot r, \leqslant 0$ & $\varepsilon 0, r V$ & $\varepsilon \vee, \wedge$. & $r \varepsilon, r r$ & $\varepsilon \varepsilon, \vee \vee$ & $\vee q, \cdot 1$ & \\
\hline$\leqslant 0,0 \mathrm{~V}$ & $\leqslant 0,90$ & $\varepsilon \wedge, \iota_{\Lambda}$ & $r_{0}, 90$ & $\varepsilon r, V T$ & $\mathrm{Vq}, 7 \mathrm{~V}$ & خوى \\
\hline$\varepsilon 7,09$ & $\varepsilon V, \cdot r$ & $\varepsilon q, \leqslant \vee$ & ro,.. & $\varepsilon 0, \vee 0$ & $\Lambda \cdot, \vee \circ$ & مرند \\
\hline$\varepsilon \vee, r V$ & $\varepsilon \vee, \wedge \vee$ & o.,$\Gamma$. & & $\leqslant 0, \varepsilon$. & $\wedge 1,0 \wedge$ & نججوان \\
\hline$\varepsilon \vee, \wedge q$ & $\Sigma \wedge, \Sigma \zeta$ & $0 \cdot, 10$ & $\Gamma_{0, \Lambda \Lambda}$ & $\leqslant \neg, r_{0}$ & $\Lambda r, \mid r$ & مراغة \\
\hline$\varepsilon \vee, \wedge q$ & $\leqslant \wedge, \leqslant\}$ & $0 \cdot, 10$ & ro, & $\varepsilon \neg,\ulcorner\wedge$ & Ar, Ir & تبريز \\
\hline$r r, \Sigma \wedge$ & $\varepsilon \wedge, \vee \wedge$ & $01, r Y$ & $r \varepsilon, r T$ & $\varepsilon \wedge, \Upsilon \wedge$ & $\wedge r, 0$. & أردبيل \\
\hline Or,Or & $0 \leqslant, \leqslant Y$ & 07,10 & $\varepsilon 1, \cdot r$ & $\varepsilon V, I T$ & $\Lambda \wedge, 1 \Gamma$ & بردعة \\
\hline r,or & $0 \leqslant, \leqslant Y$ & $07, \wedge 0$ & $\varepsilon r, r r$ & $\varepsilon \varepsilon, \wedge r$ & $\wedge \wedge, \mid \Gamma$ & تفليس (تبليس) \\
\hline $0 ., 11$ & $\circ \cdot, \vee \wedge$ & Or,Tr & $r \varepsilon, V T$ & $\varepsilon q, \vee \vee$ & $\wedge \varepsilon, 0$. & باكو \\
\hline $0 ., 11$ & $0 \cdot, \vee \wedge$ & Or,Yr & $\Gamma_{0, \wedge V}$ & $\varepsilon \wedge, \neg \uparrow$ & $\wedge \varepsilon, 0$. & اخي \\
\hline $0 \cdot, V 1$ & $01, \leqslant Y$ & or,, 0 & r, & $\varepsilon \wedge, \curlyvee \wedge$ & $\Lambda 0,1 \Gamma$ & باب الأبواب \\
\hline $00, \leqslant 1$ & $\Delta T, \leqslant Y$ & $\bullet \wedge, \wedge 0$ & $\varepsilon 1,1$. & $\varepsilon q, \cdot r$ & $q \cdot, 1 r$ & بلغار \\
\hline$\{0, \leqslant 1$ & $\varepsilon 0, \vee \wedge$ & $\Sigma \Lambda, Y Y$ & r & $\varepsilon \varepsilon, r V$ & $\vee \vee, 0$. & كوفة \\
\hline ऍ , $\leqslant \wedge$ & rᄉ, $\Sigma r$ & $\varepsilon \cdot, \wedge 0$ & TV,00 & $\varepsilon \leqslant, \circ \wedge$ & VT, Ir & مداين (مدائن) \\
\hline$\varepsilon V, r q$ & $\varepsilon \vee, \vee \wedge$ & $0 ., Y T$ & ro, vo & $\varepsilon 0, \vee 0$ & $11,0$. & واسط \\
\hline$\varepsilon 7, \cdot 1$ & $\varepsilon \neg, \leqslant r$ & $\varepsilon \wedge, \wedge 0$ & ro,v^ & $\varepsilon \varepsilon, \Gamma_{0}$ & $\Lambda \cdot, 1 \Gamma$ & بغداد \\
\hline$\varepsilon q, \vee \vee$ & $0 ., \leqslant Y$ & Or, 10 & דr, & $\varepsilon \vee, \wedge$. & $\wedge \varepsilon, 1 r$ & بصرة \\
\hline$\varepsilon q, \vee \vee$ & $0 ., \leqslant Y$ & Or,, 0 & r & $\varepsilon \vee, \vee V$ & $\Lambda \varepsilon, 1 \Gamma$ & أبلة \\
\hline $0 ., 11$ & o., $\vee \wedge$ & Or,Tr & rT, r & $\varepsilon \wedge, \curlyvee \wedge$ & $\wedge \varepsilon, 0$. & عبادان \\
\hline $0 ., 11$ & $0 \cdot, \vee \wedge$ & Or, r & To, 70 & $\varepsilon \wedge, \wedge 0$ & $\Lambda \varepsilon, 0$. & ستر (تسترَ) \\
\hline $0 ., 77$ & $01, r v$ & $o r, \wedge$. & $r ч, \Sigma T$ & $\varepsilon \Lambda,\rceil \vee$ & $\Lambda 0, \cdot \Lambda$ & أهواز \\
\hline 01,7 . & or, rV & $0 \leqslant, \wedge$. & ro, 10 & ט., & $\wedge \neg, \wedge$ & أرجان (بهبهان) \\
\hline
\end{tabular}




\begin{tabular}{|c|c|c|c|c|c|c|}
\hline$\circ 9,0 r$ & $O r, \leqslant r$ & 00,10 & $r_{0}, \Sigma \wedge$ & 01,70 & $\wedge \vee, ı$ & كازرون \\
\hline or, $9 \leq$ & or, Vᄉ & $0 Y, Y Y$ & $r \varepsilon, q r$ & Or,OV & $\Lambda \vee, o$. & فيروز ابد \\
\hline 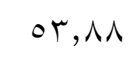 & $0 \leqslant, \vee \wedge$ & OV,YY & ro, T. & or, 9 . & $\Lambda \wedge, 0$. & إصطخر \\
\hline $0 \leqslant, \leqslant V$ & $00, \leqslant Y$ & $\bullet \vee, \wedge \circ$ & $\Gamma \varepsilon, \vee \wedge$ & $0 \leqslant, \Gamma_{0}$ & $\wedge q, 1 r$ & يزد \\
\hline$\varepsilon \wedge, r T$ & $\varepsilon \wedge, \vee \wedge$ & $01, Y Y$ & rv, 10 & $\varepsilon 0, \Gamma_{0}$ & Ar,o. & هر حلوان \\
\hline$o\{, \cdot r$ & $0 \leqslant, 90$ & 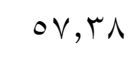 & $\varepsilon \cdot, r$. & $\Sigma \wedge, \Sigma \vee$ & $\wedge \wedge, \neg \vee$ & زنكان (زبجان) \\
\hline $0 ., 11$ & $\circ \cdot, \vee \wedge$ & Or, rr & ro, rV & $\varepsilon q, r r$ & $\wedge \varepsilon, 0$. & أبهار \\
\hline س., & $0\{, \leqslant r$ & $07, \wedge 0$ & rq, & $\varepsilon \wedge, 0$. & Nᄉ, I & همدان \\
\hline or & $01, \cdot r$ & $O r, \leqslant V$ & $r r, \wedge r$ & $0 ., 9 r$ & $\wedge \varepsilon, \vee_{0}$ & كرج \\
\hline $01, Y 1$ & 01,90 & o & $r \varepsilon, \wedge$. & $\circ \cdot \wedge \mathrm{V}$ & $10,7 \mathrm{~V}$ & قم \\
\hline $01, \wedge \varepsilon$ & or, Tr & 00,0 & $\Gamma \varepsilon, \wedge \wedge$ & $01, \leqslant 0$ & سז,1 & ري \\
\hline or, 10 & or, 90 & ه & ro,... & $01,7 \mathrm{~V}$ & $\Lambda \neg,\rceil \vee$ & إصفهان \\
\hline$r q, \varepsilon r$ & r^, $\vee \wedge$ & r & • . . & $09,0$. & $T r, 0$. & طوس \\
\hline or, vr & $0 \varepsilon, 7 r$ & $o v, .0$ & ro,91 & or, & אוץ, & آمل \\
\hline $0 q, 0 r$ & $7 \cdot, \vee \wedge$ & rr, & סץ, & 71,10 & $9 \varepsilon, 0$. & سرخس \\
\hline $00, \leqslant 1$ & $07, \leqslant Y$ & $\circ \wedge, \wedge \circ$ & ro,v. & $0 \leqslant, \leqslant$ & $q \cdot, 1 r$ & جرجان \\
\hline Yr, . & Tr, & 70,10 & rT, & $\vee r, q \wedge$ & $q \vee, 1 r$ & 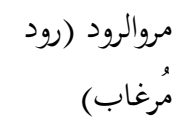 \\
\hline$q r, \varepsilon$ & $97, \leqslant r$ & $9 \wedge, \wedge 0$ & $\varepsilon 9,7 r$ & $\Lambda \cdot, o r$ & r & سرندب \\
\hline Y & $70, \wedge \wedge$ & TN,rY & rד, זr & $77,9 \mathrm{~V}$ & 99,7 . & سمرقند \\
\hline 81,91 & Vr, 90 & Vר, & $\varepsilon 1,9 r$ & $70, V T$ & $1 \cdot V, 7 V$ & ق ق ق قدهار \\
\hline$\circ V, O$. & O^, Tr & $71, \cdot v$ & ס, & $\circ \wedge, \wedge$. & or,ro & نيشابور \\
\hline r, r, & $0 \leqslant, \leqslant\}$ & $07, \wedge 0$ & ro,oo & $\circ Y, \circ \wedge$ & $\wedge \wedge, 1 \mu$ & شيراز \\
\hline$\circ 9,11$ & $\neg \cdot, \leqslant r$ & $7 r, \wedge 0$ & $r v,{ }^{\prime}$ & $07, \leqslant 0$ & $q \leqslant, \mid r$ & هرموز \\
\hline
\end{tabular}

أولغ بك, زيج الغ بك لرفاعي (تعريب: يحي

\section{المخطوطة، . (تعريج: \\ الرفاعي)، الركات}

https://dl.wdl.org/3951/service/3951.pd

f

خليفة النبهاني، ثمرة الوسيلة، مصرى: دار الكتب

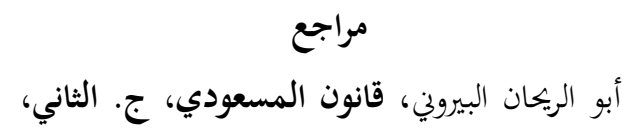

الهند: دائرة المعارف العسمانية، 1955

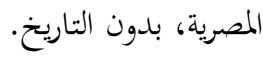


Edward S. Kennedy, Mathematical Geography, in Encylopedia of The History of Arabic Scince, Vol. I (Editet: Rashed, Roshidi and Morelon, Règis), (London: Routledge، 1996)

E. S. Kennedy and M. H. Regier, "Prime Meridians In Medieval Islamic Astronomy, Vitas in Astronomy، vol. 28، No 1 (1985) accessed 1 March 2017,doi: 10.1016/00836656(85)90005-4.

Nazir, Moh, Metode Penelitian J Jakarta: Ghalia Indonesia، 1988.

Suharsimi Arikunto، Prosedur Penelitian, Jakarta: Rineka Cipta, 2013.

$$
\begin{aligned}
& \text { سنيور كرلونلّينو، علم الفلك تاريخه عند العرب في } \\
& \text { الفرون الوسطى، بيرووت: ذو القثرقية، } 1991 . \\
& \text { شاكر خصبك، الجغرافية عند العرب، بيروت: المؤسسة } \\
& \text { العربية، 1986، الطبعة الأولى } \\
& \text { علي عبد الله, رواد علم الفلك في الحضارة العربية و } \\
& \text { الإسلامية, الرياض: الثوبة, الطبعة الثانية, } 1993 . \\
& \text { محمد منصور، سلم النيرين، كادري: الفلاح فالوصا، } \\
& \text { بدون التاريخ. }
\end{aligned}
$$

